\title{
A Proposal for an Economic Attendance Management System
}

\author{
Bokrae Jang ${ }^{1}$, Jaegeol Yim ${ }^{1, *}$ and Seung-Hyun $\mathrm{Oh}^{1}$ \\ Dept. of Computer Engineering \\ Dongguk University at Gyeongju, Gyeongbuk, Korea \\ \{pse5802,yim,shoh\}@dongguk.ac.kr
}

\begin{abstract}
This paper proposes an attendance management system (AMS) after reviewing existing AMSs. The proposed AMS uses smartphones carried by students and the wireless access points that are installed to allow Wi-Fi compliant devices such as smartphones to access to the local area network. There is almost no university campus where the local area network is not available. Since the proposed AMS does not use any other devices except the smartphones and access points, it is easy and economical to be installed. The proposed system recognizes all students in the class as attendees as long as every student carries a smartphone that is registered to the AMS. The proposed system recognizes all students who are not in the classroom as absentees. During the preparation stage, each access point is assigned a unique dynamic Internet Protocol (IP) address allocation range.
\end{abstract}

Keywords: Attendance Management System, Smartphone, Access Point, Wireless Network, Mobile Application

\section{Introduction}

Many researches to develop attendance management system (AMS) have been performed in order to automatize the time consuming traditional attendance check process. One of the key techniques used in AMS development is recognition of the students in the classroom. Biometrics, face images, radio frequency identification (RFID), the media access control (MAC) address, and Bluetooth low energy (BLE) beacons are used in student recognition. The attendance status should be eventually reflected in the grade. For example, 0.5 point is deducted per absence. Therefore, an AMS should be compatible with the learning management system.

Most of existing AMSs require some special devices to be installed in the classrooms. For example, fingerprint recognizers/RFID readers should be installed when the AMS is fingerprint-based/RFID-based. This paper proposes attendance check method that does not require any special devices dedicated for the purpose of attendance check. Nowadays all universities provide the wireless network service and every university student carries a smartphone all the time. The proposed method uses the wireless communication between a student's smartphone and the access points (AP) installed in or around the classroom.

\section{Attendance Management Systems}

Attendance information recorded in the log book should be read by the grade management computer system. It is a time consuming process to key in attendance information into the computer. Shailendra et al. [1] developed a handheld device with which a teacher can record attendance information while checking attendance. They also developed a local server that collects data from all the handheld devices through the

Received (April 27, 2017), Review Result (June 30, 2017), Accepted (July 4, 2017)

* Corresponding Author 
ZigBee technology. In the local server, a web server is also installed. They developed a web site that displays all the attendance records. One of the disadvantages of this method is that the teacher manually records attendance information on the handheld device and it will take a long if the class has many students.

Shoewu et al. [2] developed an attendance management system that has a database of students' fingerprints. Every student attending a class has to put his/her thumb on the fingerprint reader. The system automatically identifies the student matching the fingerprint and records his/her attendance. Student attendance records are stored in the database. Referring to the database, the system generates a report for each course.

Iris is well protected by cornea against external damage and is hardly deformed. Besides, a picture of an individual's iris can be taken without asking the individual to do something. Kadry and Smaili [3] proposed an attendance management system based on iris recognition. The iris recognition module is connected with a RF (radio frequency) wireless module and the attendance information is transmitted to the server. The server displays useful attendance statistics information.

Arulogun et al. [4] and Yuru et al. [5] proposed RFID-based attendance management systems. Each student carries a unique RFID (radio frequency identification) embedded into student identification card. Around the entrance of each classroom, a RFID reader is installed. The reader reads all the RFIDs within the reading range. The RFID reader is connected to the computer on which the attendance management software is running. Whenever the software receives a RFID packet, it stores the data with the read time. The read time close to the beginning/end time of the class indicates the student's arrival/leave time. After class, the software sends a message to each student notifying whether he/she has attended the class or not.

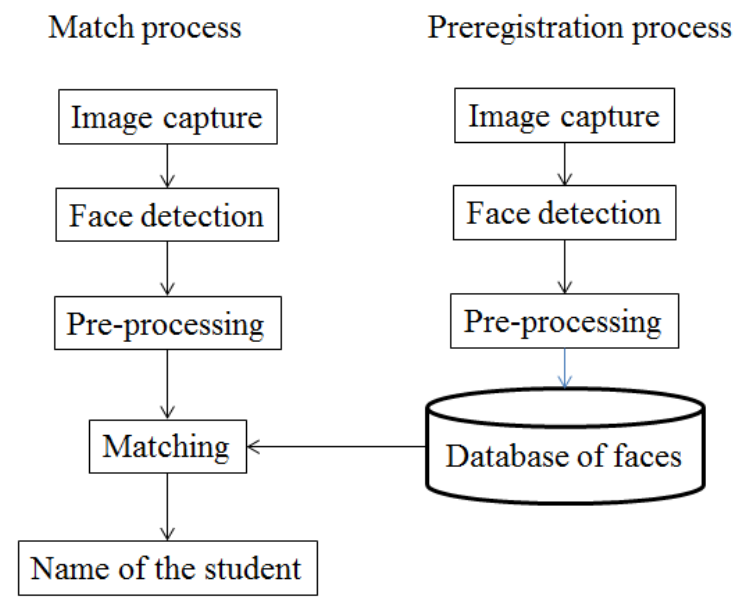

Figure 1. The Process of the Face Recognition based AMS [7]

Bhalla et al. [6] proposed a Bluetooth based attendance management system. The application running on the lecturer's smartphone initiates the process of checking attendance. Then the student's smartphone sends its Media Access Control (MAC) address to the teacher's smartphone through the Bluetooth connection. The attendance management application matches the received MAC address with the preregistered student's MAC address and confirms the presence of the student.

Chintalapati and Raghunadh [7] proposed a face recognition based attendance management system. The process of the proposed method is described in Figure 1. During the preregistration process, face images of all students are stored in the database. In the beginning of a class, the system takes a picture of each student and recognizes the name of the student. At the end of the class, the system makes a list of attendees and sends an email to an absentee. 
Shehu and Dika [8] proposed a computer vision based attendance management system. This system takes a picture of all students in the classroom with a digital camera. The system, then, detects each student's face in the picture with computer vision technology. By matching each student's face from the picture with the preregistered face, the system recognizes attended students. The list of attendees is passed to the learning management system for the further process.

Two attendance management systems were proposed in [9], one was app-based and the other was web-based. The app-based one is initiated by the app running on a student's smartphone when it sends the smartphone number to the server. The server allows the smartphone to access the wall-mounted attendance device such as beacon, RFID or QRcode when the received smartphone number is registered as a student's phone number. The app recognizes the classroom ID by reading those wall-mounted attendance devices, and sends the classroom ID along with MAC address of the smartphone to the server.

In the web-based attendance management system, the server displays a verification number on the screen in the classroom. A student should key in the number in the textbox provided by the mobile application. The client application sends the verification number, its location obtained from the Google's Positioning System, the IP address of the smartphone, and so on to the server.

Bae and Cho [10] proposed a Bluetooth low energy (BLE) Beacon based attendance check system. A beacon is installed at each classroom. When a student enters into the classroom, the attendance application running on the student's smartphone receives a beacon signal and sends the received signal with his/her identification number to the server.

Kim and Cheong [11] proposed an attendance management system that consists of a server and a client mobile application. The server system consists of a web server, an attendance management server, and the database. The client application consists of a lecturer module and a student module. The lecturer module collects AP signals and sends them to the server. The student module also collects AP signals and sends them to the server. The server compares AP signals from the lecturer module and student module. If they match it concludes that they are in the same classroom.

\section{The Proposed Attendance Management System}

This paper proposes an attendance check method that does not require any devices that are dedicated for checking attendance. The idea behind of this method is that the Internet Protocol (IP) address of a smartphone indicates the AP to which the smartphone is connected. Since the location of an AP is closely related to the classroom, we can figure out whether the owner of the smartphone is located in the classroom or not. The process of deploying the proposed AMS is illustrated in Figure 2.

The process of deploying our attendance check system consists of two phases: preparation phase and attendance check phase. During the former phase, the following operations are performed [12]:

1) For each access point (AP), assign a name indicating where the AP is installed as the service set identifier (SSID) of it, so that the name of the classroom where the router is installed can be automatically extracted. For example, if an AP is installed in "L101" classroom, then "L401" is assigned to the SSID of it.

2) For each AP, uniquely specify a dynamic IP allocation range.

3) Build a database to store relationships between smartphones, students, routers, and classes [12].

The most important component of our AMS is the mobile app. Every student and teacher must have a smartphone running this mobile app. The process of the attendance check phase consists of the following activities [12]: 
1) The teacher presents a random string as the secret string.

2) Transmitting the secret string to the server with the MAC and IP addresses and the current time.

3) The server returns acknowledge.

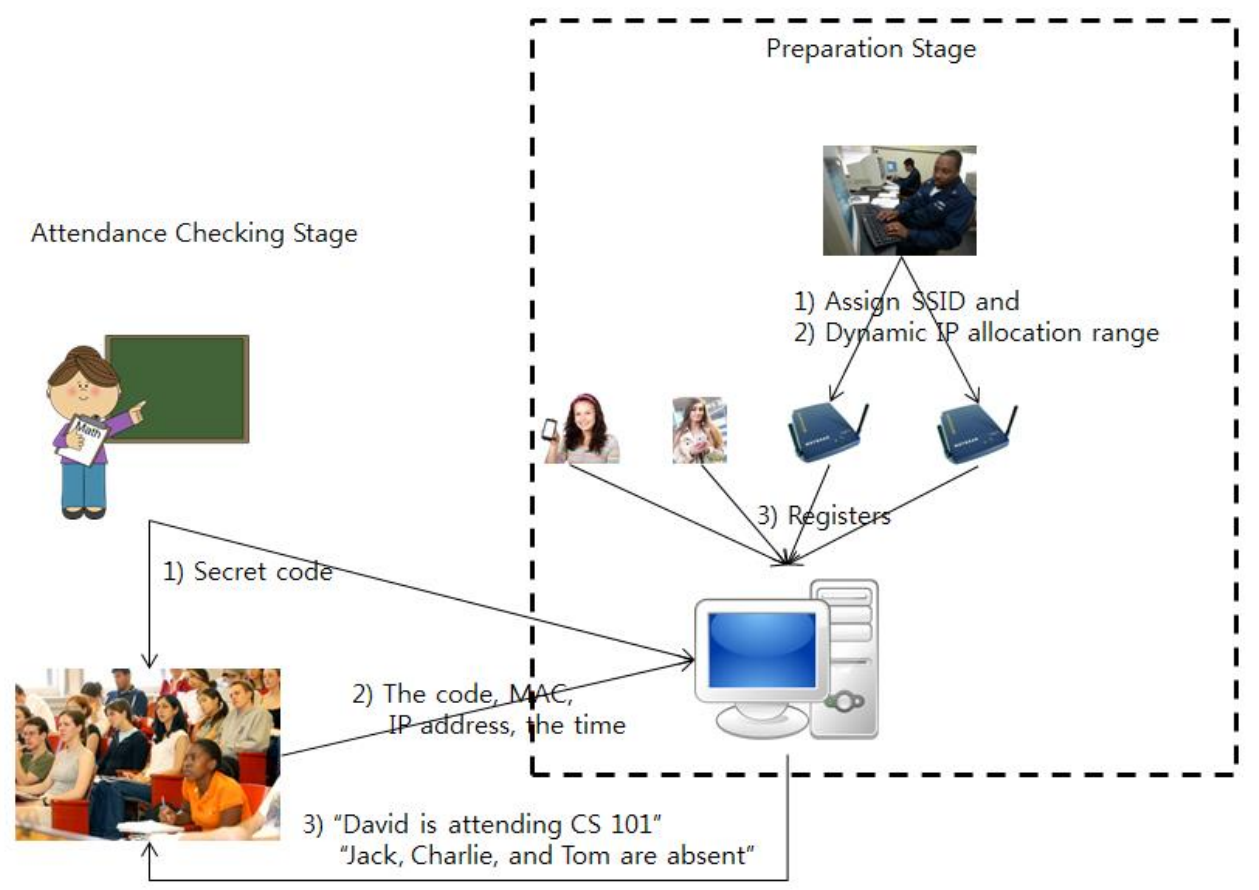

Figure 2. The Process of our AMS

A smartphone typically receives signals from multiple APs. This application offers a menu where students can specify the classroom they are attending as shown in Figure 3. A menu where the secret code can be typed in is also provided [12].

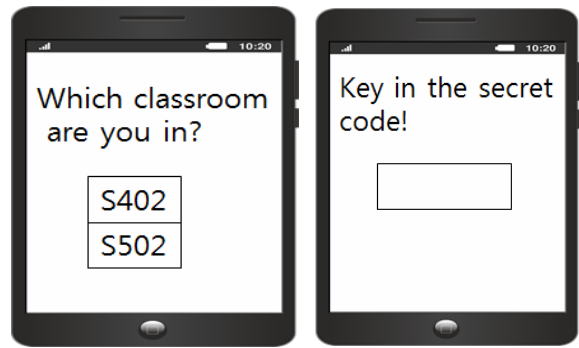

Figure 3. A Couple of Example Menus Provided by our Application [12]

\section{Design of the System}

We need a database to record information of instructors, students, schedule of classes, information of access points, and so on. Our design of the database is summarized in Figure 4. In the MeetingSC table whose primary key is MeetingSCID, the secret code, the MeetingID, and the time of the issue are recorded. For each MeetingID, there are at least one MeetingSCID. In the Present table, all students who sent the secret code that is identified by the MeetingSCID on time are recorded.

Our design of the system is described in the sequence diagram in Figure 5. The professor client has the utility class that provides methods to find the MAC address and 
the IP address of the smartphone and the MAC address of the AP. The professor client also has a communication class that transmits the message consisting of a MAC address, an IP address, and so on.

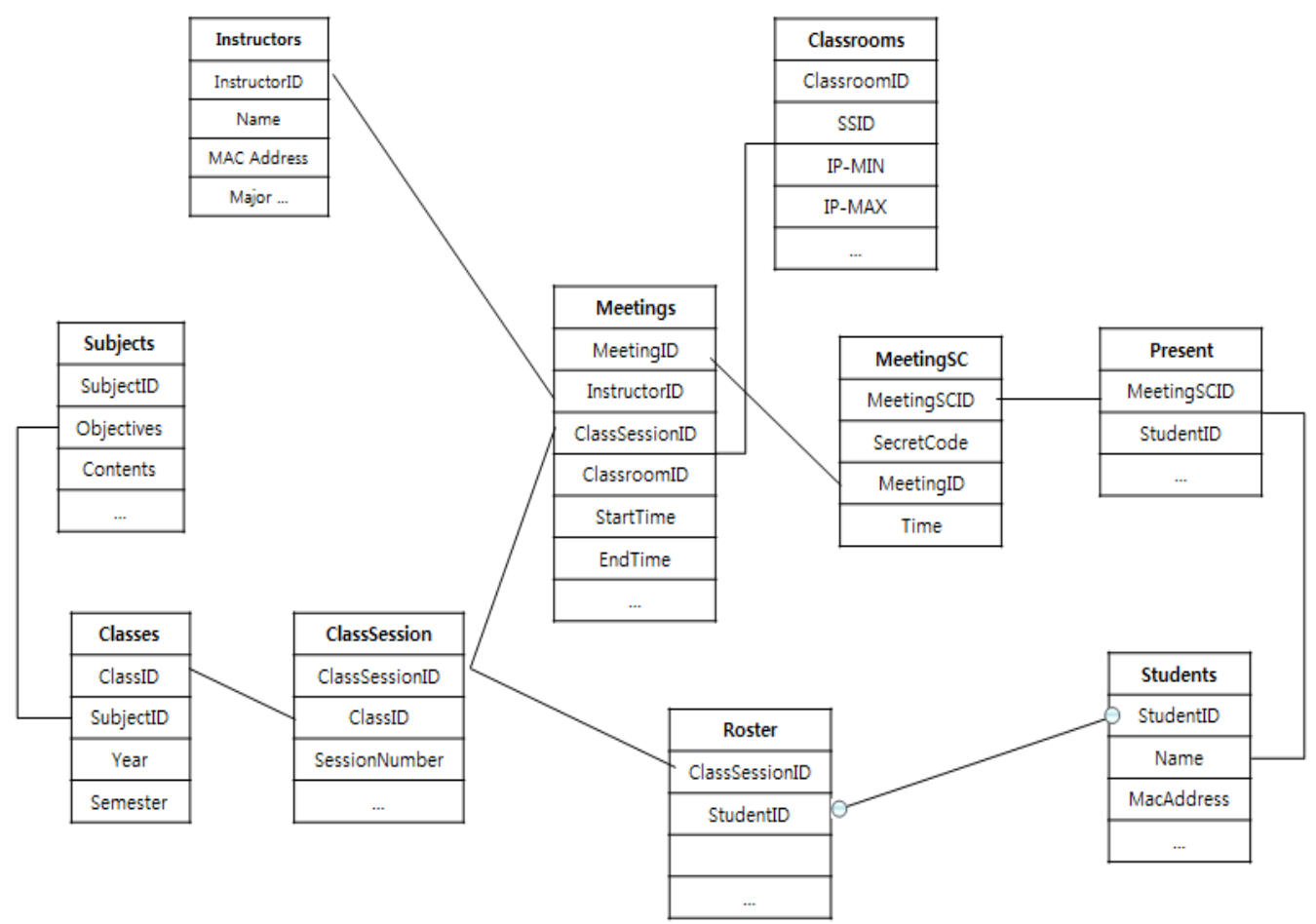

Figure 4. Part of the Database

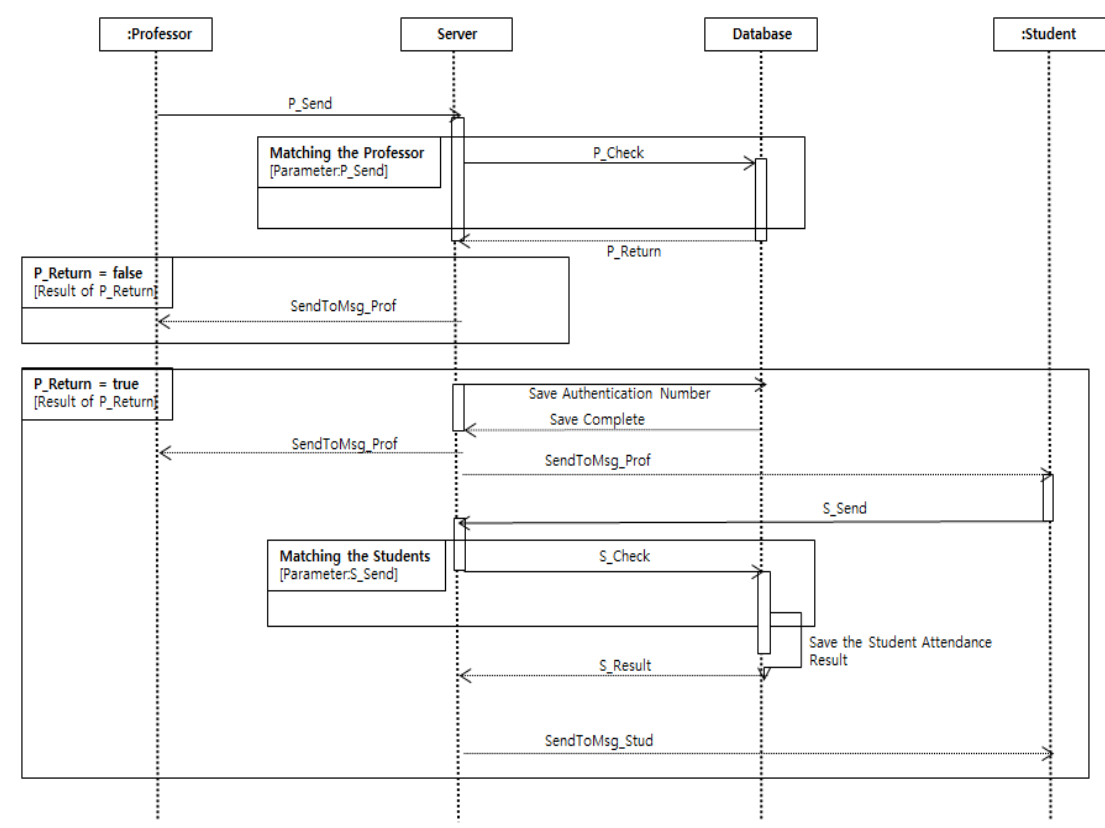

Figure 5. A Sequence Diagram Describing the Process of our System

The proposed AMS recognizes that the student in the classroom is present. A student in the classroom sends a message consisting of the MAC address, the IP address, the secret code, and others. The MAC address uniquely identifies the smartphone, and the 
smartphone uniquely identifies the student, owner of this smartphone. The system recognizes that the student identified by the MAC address is present in the class. If a student makes a mistake during keying in the secret code then the system would determine that the student is absent. Our system sends absentee messages to students who are determined to be absent. The student who keyed in the wrong secret code will send the message again so that the system recognizes him/her as an attendee. A smartphone in a classroom may receive many signals from many APs. A signal from the A AP that is installed in another classroom may happen to be stronger than the signal from the B AP installed in the classroom where the student is currently located. If the smartphone inserts the SSID of the A AP into the message, then the system would determines that the student is absent. However, our system asks students to select the SSID representing the classroom where they are located. Therefore, we conclude that the proposed AMS recognizes all attendees.

Our system determines that the students who are not in the classroom are not present. A student who are not in the classroom cannot know the secret code arbitrary created by the professor. Let's say a student in the classroom sent the secret code to his friend, S1, in the dormitory. The SSID sent by S1 would not match the SSID of the AP in the classroom. Furthermore, the IP address sent by S1 would not within the dynamic address allocation range. Therefore, this system will not determine that $\mathrm{S} 1$ is present.

We have built a hierarchical timed Petri net model for our AMS in order to verify our design. The top page of the model is shown in Figure 6. As the professor start the process, a tuple consisting of the MAC address of the professor's smartphone, the classroom ID, the IP address of the professor's smartphone, and the secret code is delivered to the server. With the MAC address and the IP address the server verifies that the issuer of the message is the professor. After verification, the server records the secret code in the database. Every student in the classroom sends a message consisting of the MAC address, IP address, the SSID, and the secret code that was typed in by the student. By investigating the messages from the students, the server determines who is present and who is absent. The tokens associated with the Msg place explicitly shows who are present and who are absent.

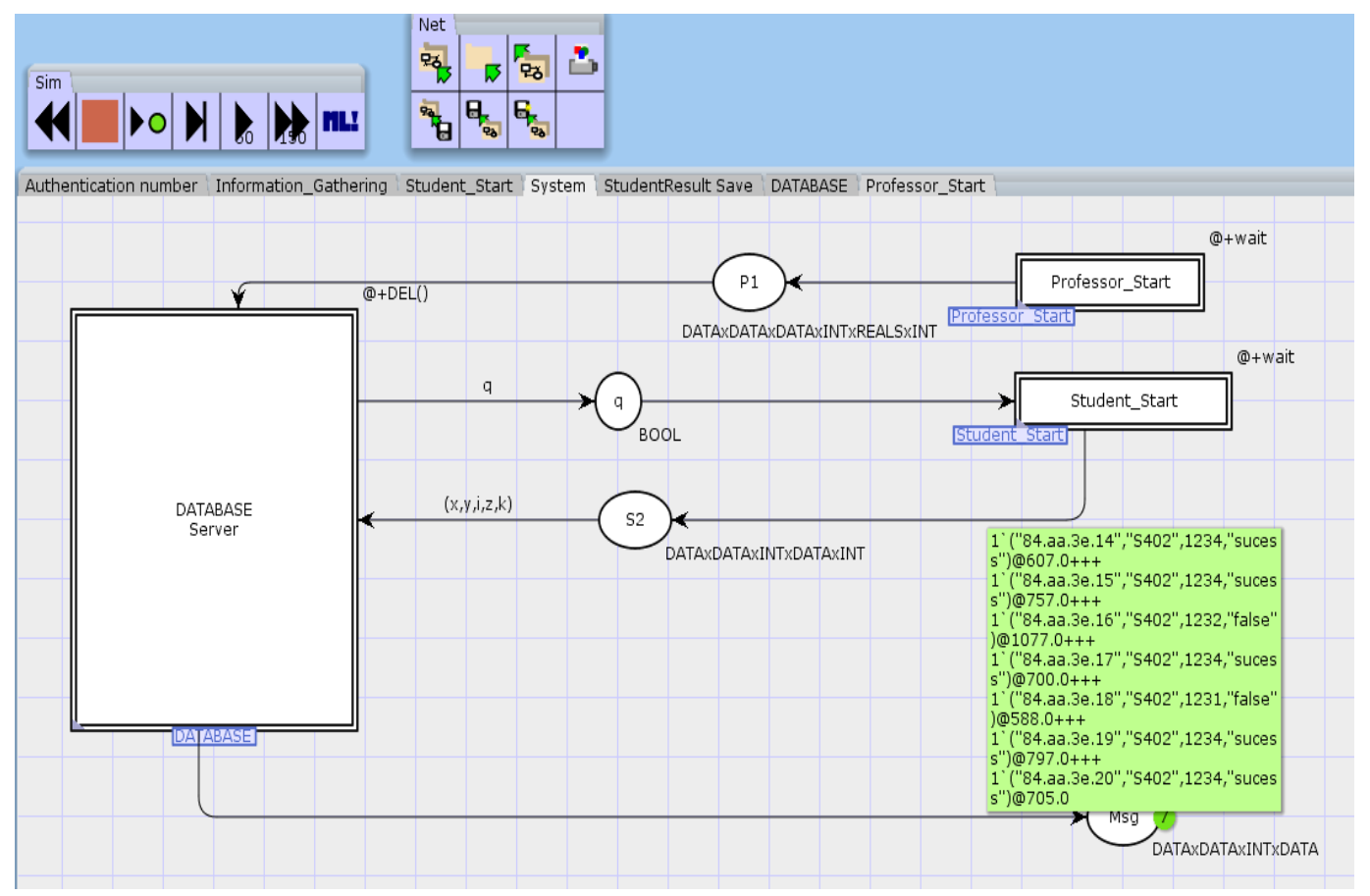

Figure 6. The Top Page of our Petri Net Model for the Process 


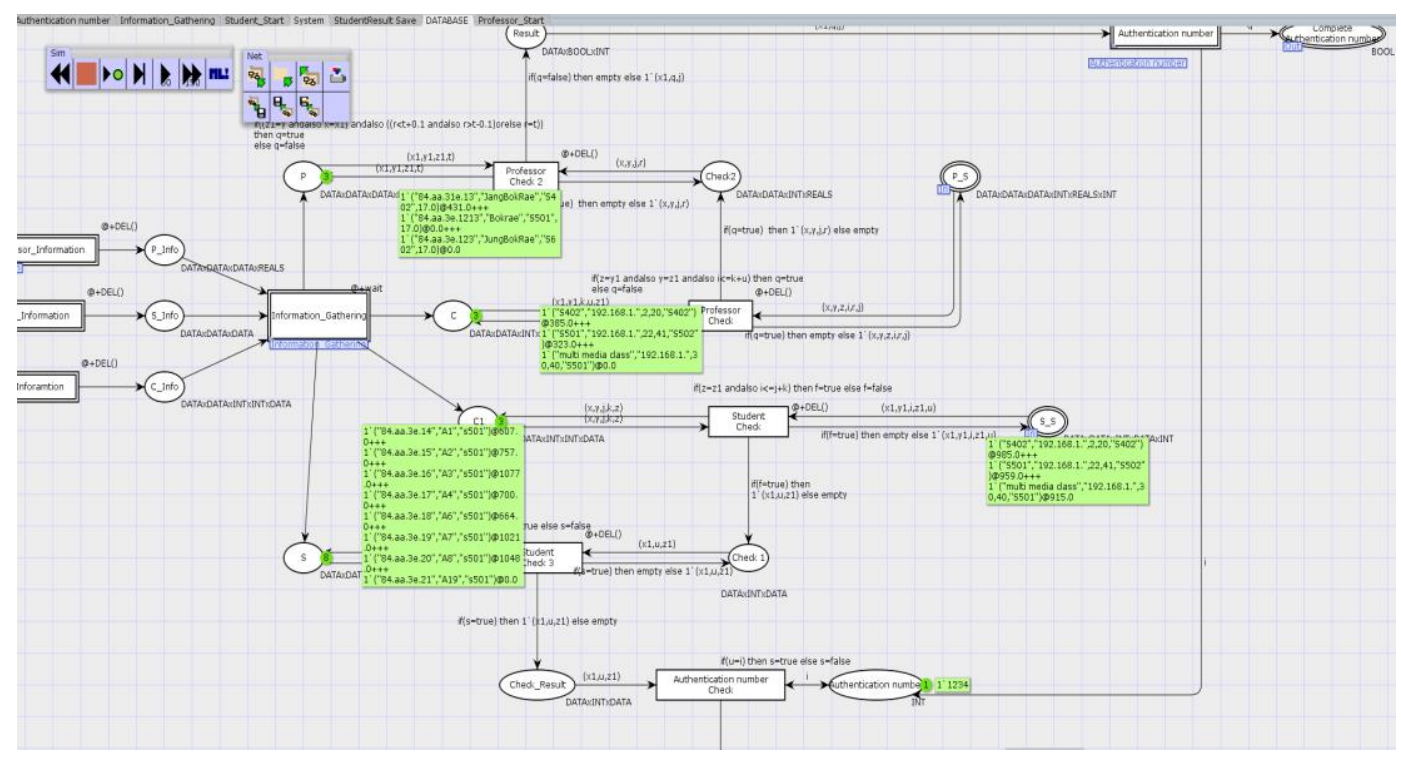

Figure 7. Part of the Process Performed by the Server

The "DATABASE Server" transition in the figure is an abstract transition representing the "DATABASE" subpage. Part of the subpage is shown in Figure 7. The "DATABASE" subpage represents the process performed by the server. For example, the "Professor Check" transition has the following guard inscription in order to verify that the MAC address in the message from the professor is the same as the MAC address of one of the registered professors' smartphones and the SSID in the message from the professor is the same as the name of the classroom where this professor has a lecture. This guard inscription also tests if the IP address in the message is within the dynamic address allocation range:

If $(\mathrm{z}=\mathrm{y} 1$ andalso $\mathrm{y}=\mathrm{z} 1$ andalso $\mathrm{i}<=\mathrm{k}+\mathrm{u})$ then $\mathrm{q}=$ true

else $\mathrm{q}=$ false

The following Java code finds the MAC address and the IP address of the Android smartphone:

WifiManager myWifiManager $=\ldots$ getSystemService $(\ldots)$;

WiFiInfo myWifiInfo = myWifiManager.getConnectionInfo();

String myMacAddress = myWifiInfo.getMacAddress();

Int myIpAddress = myWifiInfo.getIpAddress();

The following permission has to be stated in the AndroidManifest.xml:

<uses-permission android:name="android.permission.ACCESS_WIFI_STATE"/>

Using the Calendar class, the current time of the day can be found:

... Calendar.getInstance().getTime();

After collecting the MAC address, the IP address, and the MAC address of the AP, the smartphone sends the message to the server. For the communication between the server and the client applications, the Socket is used. Part of the communication server is shown in Figure 8.

After receiving a sequence of bytes with the read() method in the SocketChannel( ) class, it prints "Terminated" if the end-of-stream has been reached. Otherwise, it repeats taking off a SendData from the received string and saving the SendData in the database where SendData is a class representing a message, for example a tuple consisting of the MAC address, the IP address, the SSID, the secret code, and the time. 


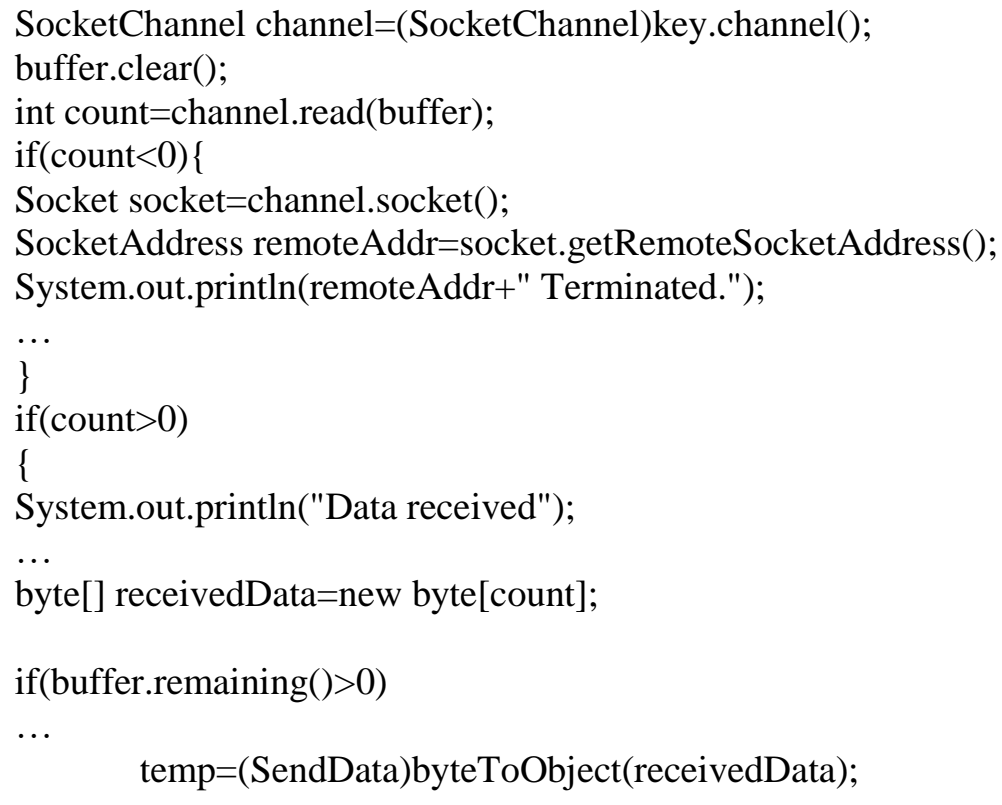

\section{Figure 8. Part of the Communication Server}

\section{Conclusion}

After reviewing existing attendance management systems (AMS), this paper proposed a new attendance check method. The proposed method is extremely convenient to use. All the students and instructors have to do is just keying in the secret string wrote down by the instructor on the blackboard. The rest of the process is done by the server application. The false negative error rate of the proposed method should be $0 \%$ as long as everybody in the classroom sends correct message to the server. A false positive result might happen if a student sends out the secret string to his/her friend, A, at home and the friend A sends the secret string using our mobile app. In this case, our server application concludes that the friend $\mathrm{A}$ is not attending the class by investigating the dynamic IP address. Therefore, the false positive error rate of the proposed method should also be $0 \%$.

The secret string sent by the student in the class might not reach the server because of network errors. The network error rate is extremely low, though. The server sends out the absent notification message to all the absent students. Therefore, the student with network error will receive the absent notification message, too. In that case, the student will resend the secret string. If the resent secret string does not reach the server again, then resending secret message will repeat until the server successfully receives the secret string. Considering the low communication error rate, we can conclude that this kind of situation would not occur in practice.

\section{Acknowledgements}

This research was supported by the Dongguk University Research Fund of 2016, by the Ministry of Education (NRF-2011-0006942), and by the Ministry of Knowledge Economy (10037393).

\section{References}

[1] S. M. Singh, A. Khan, V. Singh, A. Patil and S. Wadar, "Attendance Management System", International Conference on Electronics and Communication System, Coimbatore, India, (2015).

[2] O. Shoewu and O. A. Idowu, "Development of Attendance Management System using Biometrics", The Pacific Journal of Science and Technology, vol. 13, no.7, (2012), pp. 300-307. 
[3] S. Kadry and M. Smaili, "Wireless attendance management system based on iris recognition", Scientific Research and Essays, vol. 5, no. 12, (2010), pp. 1428-1435.

[4] O. T. Arulogun, A. Olatunbosun, O. A. Fakolujo and O. M. Olaniyi, "RFID-Based Students Attendance Management System", International Journal of Scientific \& Engineering Research, vol. 4, no. 2, (2013), pp. 1-9.

[5] Z. Yuru, C. Delong and T. Liping, "The Research and Application of College Student Attendance System based on RFID Technology”, IJCA (ISSN: 2005-4297), vol. 6, no. 2, (2013), pp. 273-282.

[6] V. Bhalla, T. Singla, A. Gahlot and V. Gupta, "Bluetooth Based Attendance Management System", International Journal of Innovations in Engineering and Technology, vol. 3, no. 1, (2013), pp. 227-233.

[7] S. Chintalapati and M. V. Raghunadh, "Automated Attendance Management System Based On Face Recognition Algorithms", IEEE International Conference on Computational Intelligence and Computing Research, Taminadu, India, (2013) December 26-28, pp. 1-5.

[8] V. Shehu and A. Dika, "Using Real Time Computer Vision Algorithms in Automatic Attendance Management Systems", Proceedings of the ITI 2010 32nd Int. Conf. on Information Technology Interfaces, Cavtat, Croatia, (2010) June 21-24, pp. 397-401.

[9] S. YuXiang, Y. Cha and C. Kim, "Comparison and Performance Analysis of App and Web-based Onestop Attendance Management”, IJUNESST (ISSN: 2005-4246), vol. 8, no. 12, (2015), pp. 325-336.

[10] M. Bae and D. Cho, "Design and Implementation of Automatic Attendance Check System Using BLE Beacon”, IJMUE (ISSN: 1975-0080), vol. 10, no. 10, (2015), pp. 177-186.

[11] J. Kim and S. Cheong, "Research on an Authentication Algorithm for an Electronic Attendance System in the Constructing of a Smart Campus", IJSIA (ISSN: 1738-9976), vol. 7, no. 6, (2013), pp. 199-208.

[12] B. Jang, J. Yim and S. Oh, "A Review of Attendance Management Systems", Advanced Science and Technology Letters (ISSN: 2287-1233), (2017), vol. 143, pp. 66-69.

\section{Authors}

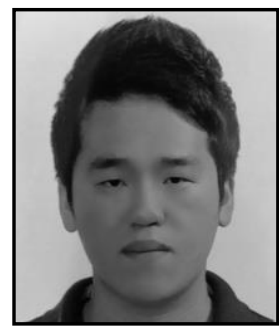

Bokrae Jang, He received an BSc. Degree in computer engineering from Dongguk University, Gyeongju, Korea, in 2015 and he is currently in the Master course of Computer Science at Dongguk University.

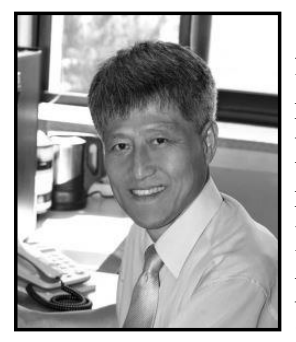

Jaegeol Yim, He received an MSc and PhD in Computer Science from the University of Illinois at Chicago, in 1987 and 1990, respectively. $\mathrm{He}$ is a Professor in the Department of Computer Engineering at Dongguk University at Gyeongju Korea. His current research interests include Petri net theory and its applications, location-based services, AI systems, and multimedia systems. He has published more than 50 journal papers, 100 conference papers (mostly written in the Korean Language), and several undergraduate textbooks.

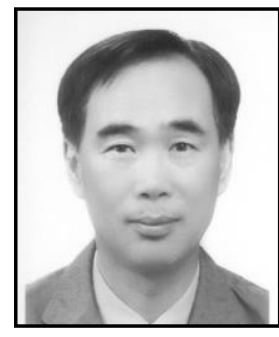

Seung-Hyun Oh, He received the B.S. degree in computer science from Dongguk University, Seoul, Korea, in 1988, and the M.S. and $\mathrm{Ph} . \mathrm{D}$. degrees in computer engineering from Dongguk University, Seoul, Korea, in 1998 and 2001, respectively. He has been a Professor with the Department of Computer Engineering, Dongguk University Gyeongju Campus since 2002. His current research interests include wireless communications, sensor networking and smart home network. 
International Journal of Database Theory and Application Vol.10, No.9 (2017) 